\title{
Lack of in vitro resistance of Candida albicans to ketoconazole, itraconazole and clotrimazole in women treated for recurrent vaginal candidiasis
}

\author{
I W Fong, R M Bannatyne, P Wong
}

\begin{abstract}
Objective-To determine whether in vitro resistance of Candida albicans to the imidazoles (ketoconazole, clotrimazole and itraconazole) is associated with recurrence of candida vaginitis.

Design-Candida isolates were collected before, during and after treatment from women with recurrent vaginal candidiasis ( $\geqslant 4$ episodes/year), randomised into two prospective studies: (1) 56 women treated with ketoconazole $400 \mathrm{mg} /$ daily for 7 days; (2) 44 women randomised to receive itraconazole $200 \mathrm{mg}$ orally, or clotrimazole $200 \mathrm{mg}$ intravaginally, twice weekly for six months.

Setting-Women's Candida Clinic at St. Michael's Hospital, a University of Toronto teaching Hospital, Toronto, Ontario, Canada.
\end{abstract}

Main outcome, measures-Isolates of yeasts recovered pre and post treatment were tested for significant changes in $\mathbf{5 0 \%}$ inhibitory concentration $\left(\mathrm{IC}_{s 0}\right)$. Resistance was defined as a greater than fourfold increase in baseline $\mathrm{IC}_{\text {so }}$ of post treatment isolates compared with pretreatment isolates.

Results-Over 250 strains of $C$ albicans were tested and none showed development of resistance to any of the agents.

Conclusion-Recurrence of vaginal candidiasis is not related to the development of drug resistance.

(Genitourin Med 1993;69:44-46)

\section{Introduction}

The pathogenesis of recurrent candida vulvovaginitis in patients with no obvious predisposing factors is poorly understood, ${ }^{1}$ and the reasons for the refractoriness of the condition after discontinuation of therapy ${ }^{2}$ is unknown. Whether the recurrences of infection represent relapse or reinfection is also undetermined. ${ }^{3}$ True vaginal relapse due to incomplete eradication of the initial vaginal infection may be responsible for a large percentage of recurrent episodes $;{ }^{4}$ however, the reason for the particular vulnerability of these women to recurrent symptomatic infection is unclear. The possibility of persistence of infection due to development of drug resistant strains exists, but there are little data on the antifungal susceptibility of Candida albicans recovered from women with this condition. Recent reports of the development of in vitro resistance to ketoconazole and fluconazole in patients with the acquired immunodeficiency syndrome (AIDS) and oropharyngeal candidiasis, ${ }^{56}$ underscores the importance of this possibility in women with chronic recurrent vaginal candidiasis as they are also exposed to long term chronic treatment with antifungal agents.

\section{Methods}

Patient population: Isolates of Candida spp were collected from patients randomised into two prospective studies. Healthy women between the ages of 18-65 years with documented (culture proven) recurrent vaginal candidiasis $(\geqslant 4$ episodes per year), with no chronic underlying diseases (such as diabetes mellitus, immunodeficiency syndrome, etc), nor receiving any immunosuppressive medications or chronic antibiotics, were studied. These studies were approved by the hospital's Human Subjects Committee and written consent was obtained from each patient.

In study (A) all patients (women) were treated with ketoconazole $400 \mathrm{mg}$ daily for 7 days, and half the male partners were treated, to assess the value of treating the sexual partners. In study (B), women were randomised to receive two forms of chronic suppressive therapy: (i) Itraconazole $200 \mathrm{mg}$ orally daily for 5 days, then twice weekly for 6 months; or (ii) Clotrimazole $200 \mathrm{mg}$ intravaginally daily for 5 days, then $200 \mathrm{mg}$ twice weekly for 6 months. At the beginning of each study, all patients were suffering from acute symptomatic vulvovaginal candidiasis that was confirmed by both direct microscopy and culture. At the initial and subsequent visits, specimens were obtained by sterile cotton swabs from the vulva, vagina, rectum and oral cavity. Washings of the vagina, for quantitative cultures, were also obtained following irrigation with $5 \mathrm{ml}$ of sterile saline. Repeat cultures from all four sites were obtained 1-2 weeks after the initiation of therapy, and monthly, thereafter, for a total of 12 months. Additional cultures were obtained if any patient developed a symptomatic recurrence of vaginitis. All specimens were inoculated onto Sabouraud glucose agar plates, and vaginal specimens were also inoculated onto chocolate agar. Typically-appearing colonies of candida growing on agar plates were Gram-stained to verify their identity as yeasts. Isolates were identified as Candida albicans by the presence of germ tube production in human serum and the production of chlamydospores in corn meal agar. Coded isolates of $C$ albicans were stored in $40 \%$ glycerol at $-70^{\circ} \mathrm{C}$ and subcultures to 
Table Susceptibility of candida isolates (IC $C_{\text {so }}$ for $90 \%$ ) before, during and after therapy

\begin{tabular}{lllll}
\hline & $\begin{array}{l}\text { Before } \\
(n=110) \\
I C_{s o} m g / l\end{array}$ & $\begin{array}{l}\text { During } \\
(n=50) \\
\mathrm{IC}_{50} \mathrm{mg} / \mathrm{l}\end{array}$ & $\begin{array}{l}\text { After } \\
(n=94) \\
I C_{s o} m g / l\end{array}$ & Range \\
\hline Ketoconazole & 0.04 & 0.04 & 0.04 & $0.02-0.63$ \\
Itraconazole & 0.04 & 0.02 & 0.04 & $0.02-0.04$ \\
Clotrimazole & 0.04 & 0.04 & 0.04 & $0.02-0.08$ \\
\hline
\end{tabular}

Sabouraud dextrose agar at $35^{\circ} \mathrm{C}$ for 48 hours prior to susceptibility testing. Isolates were tested blind and in duplicate.

Antifungal Agents: Pure powders of ketoconazole and itraconazole (Janssen Pharmaceuticals, Belgium), and clotrimazole (Miles Laboratories, Toronto, Canada), were dissolved in $100 \%$ dimethylsulfoxide to provide master dilutions of $1000 \mathrm{mg} / \mathrm{l}$ which were stored at $-70^{\circ} \mathrm{C}$.

Susceptibility Testing Procedure: A broth macrodilution method $^{7}$ was utilised with modification to improve endpoint reading. ${ }^{8}$ Inocula were prepared from a suspension of yeast colonies in sterile water containing $0.05 \%$ tween 80 which was vortexed and adjusted spectrophotometically $(90 \%$ transmittance at $530 \mathrm{~nm}$ ) to correspond to $10^{6}$ colony forming units $/ \mathrm{ml}$ by viable count. Fifty microlitres (final concentration $2.2 \times 10^{4} \mathrm{cfu} / \mathrm{ml}$ ) were added to twofold dilutions of the drugs ranging from $0.02-10.0 \mathrm{mg} / 1$ in yeast nitrogen broth. Some strains of yeasts were also tested in RPMI-1640 media for comparison. Drug/ organisms suspensions were incubated at $35^{\circ} \mathrm{C}$ for $\mathbf{4 8}$ hours. Following vortexing to obtain an even suspension, percentage transmittance at $530 \mathrm{~nm}$ was determined spectrophotometically. The inhibitory concentration was obtained by determining the lowest drug concentration at which $50 \%$ inhibition occurred compared with a drug free control. The $50 \%$ inhibitory endpoint concentration $\left(\mathrm{IC}_{50}\right)$ was defined as the lowest drug concentration satisfying the equation $\% \mathrm{~T}>\mathrm{T}_{\mathrm{c}}+1 / 2\left(100-\mathrm{T}_{\mathrm{c}}\right)$ where $T$ is the transmittance of the test and $T_{c}$ the transmittance of a drug-free control. ${ }^{8}$ Isolates obtained pre-, per- and post-therapy from the same patients were usually run on the same day. Resistance was defined as a greater than four-fold increase in baseline $\mathrm{IC}_{50}$ of post treatment isolates compared to pre-treatment isolates.

\section{Results}

Two hundred and fifty isolates of $C$ albicans, and four isolates of non- $C$ albicans strains were recovered and tested. Of the 110 candida isolates obtained before treatment, 90 were recovered from the vagina or vulva, 15 from the rectum and five from the mouth. Fifty candida isolates were obtained during treatment and of these, 40 were recovered from the vagina/vulva, seven from the rectum and three from the mouth. Of the 94 candida isolates obtained after treatment, 78 isolates were from the vagina/vulva, 10 from the rectum and two from the mouth. No significant difference in the results was observed between the two media tested. The range of $\mathrm{IC}_{50}$ values was confined to a relatively narrow range at the low end of concentrations for all three drugs (table). There was no significant changes in the IC $_{50}$ for any of the drugs tested for $90 \%$ or more of isolates obtained before, during or after therapy (table). Only one isolate obtained from the vulva showed a significant increase in the $\mathrm{IC}_{50}$ post-therapy over baseline, from $0.02 \mathrm{mg} / 1$ to $0.63 \mathrm{mg} / 1$. However, this was considered an aberrant result as the isolates from the vagina and elsewhere did not show greater than four fold increase in $\mathrm{IC}_{50}$.

\section{Discussion}

Recurrent vaginal candidiasis could possibly result from a clinically inapparent focus in the vagina. Vaginal relapse due to failure to eradicate the yeast with therapy despite clinical relief of symptoms has been postulated. Even though post therapy cultures are often negative, with clinical improvement, a single vaginal swab may be inadequate to detect low numbers of organisms, ${ }^{9}$ and may not confirm total elimination of yeast from the vagina. It is also unclear whether superficial invasion of yeast cells may result in the transition to an intracellular phase of the yeast with subsequent re-emergence weeks later as recolonization and infection of the vagina. Another popular theory to explain recurrences is reinfection from an intestinal reservoir, ${ }^{10}$ However, drugs such as ketoconazole and itraconazole should theoretically eradicate organisms from the intestinal reservoir and vaginal epithelial cells. Thus, testing for the development of in vitro resistance to explain persistent colonisation and reinfection could provide important information.

AIDS patients with recurrent oropharyngeal candidiasis have a somewhat similar problem, requiring long term suppressive therapy to prevent recurrences. Recent reports ${ }^{56}$ have documented the development of in vitro resistance associated with clinical failure to both ketoconazole and fluconazole.

Our study has shown that development of in vitro resistance to imidazoles in women treated with long term suppressive therapy is usually not seen. This is similar to the previous report of Sobel ${ }^{11}$ where increased resistance to ketoconazole was not found in a similar group of patients, although only 40 isolates were tested. Similarly, Takada et al ${ }^{12}$ noted no increased resistance to clotrimazole, although there was relative increase in recovery of Torulopsis glabrata with MIC values four times as high as those against $C$ albicans. While there is a general lack of correlation between in vitro sensitivity and invivo response with the imidazoles, ${ }^{13}$ recent reports in AIDS patients as previously discussed have found a correlation with in vitro resistance and clinical failure or recurrences.

The value of in vitro testing of fungi has been questioned also because of lack of standardisation of the method and inter-laboratory variation. ${ }^{14}$ However, although there is lack of reproducibility of results between laboratories 
testing the same organisms, the reproducibility within the same laboratory is generally consistent. ${ }^{14}$ The method chosen in our study has overcome some of the concerns in antifungal susceptibility testing, such as trailing endpoints with subjective variability in the reading of fine haze, ${ }^{15}$ and also the results are independent of inoculum size. ${ }^{8}$ Moreover the relatively narrow range of inhibitory concentrations and lack of significant changes between isolates obtained before and after therapy speak for themselves. Although there may be difficulty and controversy in interpreting isolated fungal susceptibility results, our results with measurement of IC50 from isolates obtained from the same patient at different times and performed on the same day should be more reliable.

In summary there is no evidence that development of drug resistance plays a role in recurrent vulvovaginal candidiasis.

This work was presented in part at the 31 st Interscience Conference on Antimicrobial Agents and Chemotherapy, 29 September-2 October 1991, Chicago, Ill, USA.

This study was supported by a grant from Janssen Pharmaceutica Inc, Mississauga, Ontario. We thank Mrs D Bajhan for her assistance in preparing this manuscript.

1 Sobel JD. Epidemiology and pathogenesis of recurrent vulvovaginal candidiasis. Am $\mathcal{f}$ Obstet Gyneacol 1985; 152:924-35.

2 Sobel JD. Vulvovaginal candidiasis-what we do and do not know. Ann Intern Med 1984;101:390-2.
3 Bushnell TEC, Evan EGV, Meaden JD, Milne JD, Warnock DW. Intermittent local prophylaxis against recurrent vaginal candidiasis. Genitourin. Med 1988;62:335-8.

4 O'Connor MI, Sobel JD. Epidemiology of recurrent vulvovaginal candidiasis: Identification and strain differentiavaginal candidiasis: Identification and strain differentia-

5 Tavitian A, Raufman JP, Rosenthal LE, Weber J, Webber CA and Dinesoy HP. Ketoconazole-resistant candida esophagitis in patient with acquired immuno-deficiency syndrome. Gastroenterology 1986;90:443-5.

6 Fox R, Neal KR, Leen CLS, Ellis ME, Mandal BK. Fluconazole resistant candida in AIDS. $\mathcal{f}$ Infection 1991;22:201-3.

7 Shawomy F, Espinel-Ingroff A, Cartwright RY. Laboratory studies with antifungal agents. In: Lennett EH, Spaulding EH, Truant JP (eds). Manual of Clinical Microbiology, 4th ed. Washington DC. American Society for Microbiology, 1985:991-5.

8 Galgiani JN, Stevens DA. 1978. Antimicrobiol susceptibility testing of yeasts: trubidometric technique independent of testing of yeasts: trubidometric technique independent of inoculum

9 Odds FC. Genital candidiasis. Clin Exp Dermatol 1982; 7:345-54.

10 Miles MR. Olsen L, Rogers A. Recurrent vaginal candidiasis. Importance of an intestinal reservoir. $\Im A M A$ 1977;238:1836-7.

11 Sobel JD. Management of recurrent vulvo-vaginal candidiasis with intermittent ketoconazole prophylaxis. Obstet Gynecol 1985:65:435-40.

12 Takada M, Kubata T, Hogaki M, Okawi T, Uchida K, Yamaguchi $\mathrm{H}$. Attributes of microorganisms that contribute to recurrence and intractability of vaginal mycosis. Acta Obstet Gyneacol (Japan) 1986:38;1125-34.

13 Saag MS and Dismukes WE. Azole antifungal agents: Emphasis on new triazoles. Antimicrob Agents Chemother Emphasis on

14 Galgiani JN, Reiser J, Brass C, Espanel-Ingroff A, Gordon MA, Kerkering TM. Comparison of relative susceptibility of Candida species to three antifungal agents as determined by unstandardized methods. Antimicrob Agents Chemother 1987:31;1343-7.

15 Hughes CE, Bennett RL, Beggs WH. Broth dilution testing of Candida albicans susceptibility to ketoconazole. Ant microb Agents Chemother 1987:31:643-6. 